\title{
Limnología de sistemas experimentales de lagunaje para el tratamiento de aguas residuales.
}

\author{
González, J.M.; Bécares, E. y Luis, E. \\ Área de Ecología, Facultad de Biología. Universidad de León \\ Campus de Vegazana s.n. 24071, León (España).degjgm@unileon.es
}

\section{RESUMEN}

En el presente trabajo se ha estudiado las características biológicas y físico-químicas de dos sistemas de tratamiento a escalapiloto basados en el desarrollo de microalgas: el lagunaje natural y el lagunaje de alta carga. El lagunaje natural imita los procesos característicos de una laguna hipertrófica en la que el gradiente vertical organiza las comunidades y los procesos de interés para el tratamiento del agua. El lagunaje de alta carga sin embargo mantiene la biomasa en constante turbulencia por lo que no existe gradientes verticales y la densidad de algas puede ser mucho mayor que en el primero, lo que favorece una menor demanda de espacio y la capacidad de tratar aguas muy cargadas. Ambos sistemas fueron estudiados durante dos años, tratando el mismo agua residual. Los resultados han revelado una mayor eficacia en la eliminación de materia orgánica y mayor producción de algas por el lagunaje de alta carga. Los balances de nutrientes reflejan la gran capacidad para eliminar nitrógeno y la influencia del tiempo de retención y el pH del sistema. Las cloroficeas (Monoraphidium sp.) dominaron el sistema de alta carga mientras que en el lagunaje convencional la dominancia estuvo repartida entre cloroficeas (Chlorella sp.) y criptofíceas (Cryptomonas sp.).

Palabras clave: Agua residual, materia orgánica, nutrientes, sistemas fotosintéticos lagunaje convencional, lagunaje de alta carga.

\begin{abstract}
Biological and physico-chemical characteristics of water treated with two pilot-scale treatment systems based on the development of microalgae were studied. One was a natural pond, the other, a High Rate Algae Pond (HRAP). The natural pond mimics typical processes occurring in hypertrophic waterbodies, where vertical gradients in most limnological variables, organise the biological communities and the processes of interestfor the treatment of the water: The High Rate Algae Pond, on the other hand, maintains biomass in constant turbulence. Thus, vertical gradients do not exist and, on the other hand, algal density can be much greater than in thefirst system, favouring a smaller demandfor space by the algae and a higher capacity to process heavily loaded waters. Both systems were studied during two years, treating the same type of wastewater: Results show the HRAP eliminates organic matter more efficiently than the natural lagoon, and promotes greater algae production. The balance of nutrients also reflects the great capacity to eliminate nitrogen of the HRAP and the influence of retention time and $\mathrm{pH}$ of the system on depuration efficiency. The chlorophyte Monoraphidium sp. dominated the high-rate system, while in the natural pond both the chlorophyte Chlorella sp. and the cryptophyte Cryptomonas sp. were abundant.
\end{abstract}

Keywords: Wastewater, municipal sewage treatment, organic matter and nutrients removal, photosyntetic system, High Rate Algal Pond (HRAP), Waste Stabilization Pond

\section{INTRODUCCIÓN}

La acción humana y concretamente el vertido de agua residual bruta sobre el medio natural causa una elevada degradación sobre la red hidrográfica. De esta forma, la eutrofización y la asfixia del medio acuático provocan la muerte biológica de ciertos cursos de agua.

El agua residual es una potencial fuente de energía que en muchos casos se desaprovecha debido principalmente a problemas técnicos e incluso de gestión. El grado de depuración del 
agua residual dependerá en gran medida del aprovechamiento a que vaya a ser sometida. Algunas tecnologías de bajo coste están especialmente indicadas para el tratamiento de aguas residuales destinadas a ser reutilizadas, tal es el caso de la técnica del lagunaje cuyos efluentes presentan una concentración muy baja de microorganismos patógenos, lo que resulta idóneo para su utilización agrícola, (Sierra y Peñalver, 1989; Mara y Cairncross, 1990; Mujeriego, 1990)

En España existen numerosos núcleos de pequeña y baja densidad de población sin ningún tipo de estación depuradora (el $93 \%$ de las poblaciones con menos de 10.000 habitantes según el informe de la Dirección General de Calidad de Aguas a la Comisión Delegada del Gobierno para asuntos económicos sobre la depuración de aguas residuales urbanas en España y las implicaciones de la aplicación de la Directiva 91/271/CEE, MOPT, 1992) y aquellos que la poseen sufren mayoritariamente el mal funcionamiento de las mismas o bien los tratamientos aplicados no resultan adecuados a las características de la zona, sin haberse tenido en cuenta la reducción y minimización de los impactos negativos que la carga de nutrientes $(\mathrm{N}, \mathrm{P})$ produce en los cuerpos receptores, como es el caso de las áreas definidas como sensibles.

La viabilidad económica es un problema acuciante en la instalación y mantenimiento de plantas de tratamiento de aguas residuales, por lo que se hace necesario desarrollar nuevas tecnologías de bajo coste y mantenimiento que permitan además de la eliminación de la carga contaminante y/o la eliminación de nutrientes, la posible rentabilización de las plantas de tratamiento de aguas residuales con la reutilización de los efluentes regenerados y el aprovechamiento de la biomasa obtenida por producción biológica, circunstancias éstas que no suelen darse en los procedimientos de tratamiento considerados como convencionales.

Las plantas de depuración por lagunaje se encuentran dentro de los tratamientos que sin perder eficiencia se conforman como solución económica. No obstante, al tratarse de sistemas biológicos dependientes de condiciones ambientales, es necesaria una planificación para situaciones concretas, fundamentalmente las condiciones climáticas que interaccionan con los componentes biológicos del sistema.

Los sistemas de tratamiento de aguas residuales en los que se desarrollan microalgas han sido particularmente atractivos debido a la capacidad fotosintética para utilizar la luz solar y producir el oxígeno necesario para la degradación biológica de la materia orgánica y su transformación en biomasa utilizable. Además de la utilización directa de los nutrientes (asimilación mixótrofa de la materia orgánica o autótrofa del carbono inorgánico), las algas también intervienen en la reducción de nutrientes por procesos derivados de la actividad fotosintética, como son la precipitación de fosfatos o el "stripping" del amonio como consecuencia del elevado $\mathrm{pH}$. En estos sistemas se reconoce también su capacidad antibacteriana o desinfectante, disminuyendo notablemente la propagación de bacterias patógenas al medio receptor (Sierra y Peñalver, 1989; Mara y Cairncross, 1990).

Hasta ahora en España, los sistemas de lagunaje más utilizados han sido los anaerobios o facultativos profundos (aunque no con mucha fortuna debido a múltiples razones), junto a las lagunas más someras de estabilización o lagunaje convencional, cuyas dos características principales son su bajo coste de inversión y mantenimiento y la necesidad de grandes superficies de terreno. Se añaden a las anteriores otras dos peculiaridades hasta ahora poco desarrolladas en nuestro país como son la reutilización de los efluentes en la agricultura y la potencial recuperación de la biomasa producida para su comercialización. Sobre esta base, se asocian dos circunstancias problemáticas en el lagunaje convencional. Estas son: la necesidad de terrenos (no siempre disponibles en determinadas áreas) y la dificultad de separar las algas de la fase líquida del efluente.

El sistema de lagunaje de alto rendimiento "High Rate Algae Pond" (HRAP) se adapta como una solución a los planteamientos anteriormente descritos, ya que con una mínima inversión económica, se consigue no sólo la depuración 
hasta los mejores niveles de calidad conocidos, sino también la producción de biomasa de forma rentable y fácilmente extraible de la fase líquida.

No se tiene constancia de que este sistema de HRAP haya sido aplicado a escala real en España. Tan sólo se conocen estudios a escala experimental (García, J, 1996) y elaborados en la Universidad de Barcelona, si bien desde los años 50 en que fueron inicialmente descritos (Oswald \& Gotaas, 1957), se han aplicado en diferentes partes del mundo (Oswald, et al, 1957; Azov \& Shelef, 1982; Shelef \& Azov, 1987, Shelef, 1987).

Un H.R.A.P. es un sistema único que consigue alcanzar dos objetivos: el tratamiento de las aguas residuales y la producción de biomasa, ambos con una inversión mínima y una notable reducción de espacio. Funcionalmente se trata de una combinación entre un lagunaje convencional (proceso fotosintético) y un reactor de algas de flujo-pistón (mezcla continua), persiguiendo la selección de especies con máxima tasa de eliminación de nutrientes y la máxima capacidad de floculación de forma que su posterior eliminación pueda realizarse gracias a una simple decantación.

\section{MATERIAL Y MÉTODOS}

El estudio se llevó a cabo durante un periodo de 2 años en el campo experimental de depuración de bajo coste ubicado en la localidad de Mansilla de las Mulas (León), una población eminentemente agrícola de 1650 habitantes, emplazada en el cuadrante suroriental de la provincia.

El tipo climático en la zona es Mediterráneo Templado (Papadakis. 1961), caracterizado por unos niveles anuales de precipitación de 500 $\mathrm{mm}$ aproximadamente, una temperatura media anual de 11 "C y una temperatura media de las mínimas de $-1,1{ }^{\circ} \mathrm{C}$.

Dos son los sistemas experimentales ensayados para el estudio, lagunaje convencional y lagunaje de alta carga (HRAP). El sistema convencional consiste en un estanque rectangular de $11.015 \mathrm{~m}^{3}$, una profundidad de $73 \mathrm{~cm}$ y una superficie de $15.09 \mathrm{~m}^{2}$. El sistema de alta carga consiste en un estanque somero de $30 \mathrm{~cm}$ de profundidad, a fin de no limitar la penetración de la luz (Oswald, 1988), con un volumen de 4621 y una superficie de $1.54 \mathrm{~m}^{2}$. En este último sistema el agua se encuentra permanentemente agitada gracias a un mecanismo de aspas que con una velocidad de rotación de 3 r.p.m. transmiten al líquido de mezcla una velocidad de translación de $15 \mathrm{~cm} / \mathrm{s}$, óptima según las indicaciones de Oswald, 1988.

El agua residual que abastece a los sistemas se toma directamente del colector de la población que vierte finalmente en el río Esla. El tiempo de retención hidráulico se mantuvo constante en el

Table 1. Tiempos de retención hidráulicos ensayados (TRH) y cargas medias aplicadas a los sistemas de HRAP y Lagunaje durante el estudio. Hydraulic retention times tested (HRT) and mean loads applied to HRAP and natural systems during the study period.

\begin{tabular}{lcccc}
\hline & & HRAP & & LAGUNAJE \\
\hline TRH teórico (días) & 10 & 5 & 3 & 20 \\
TRH real medio (días) & 9.65 & 4.37 & 3.33 & 24.13 \\
\hline CARGAS SUPERFICIALES: & & & & 0.57 \\
g SST $/ \mathrm{m}^{2} / \mathrm{d}$ & 0.96 & 1.22 & 1.74 & 2.48 \\
g DQO $/ \mathrm{m}^{2} / \mathrm{d}$ & 3.39 & 6.23 & 7.12 & 0.63 \\
g DBO5/m²/d & 0.88 & 1.47 & 1.92 & 0.52 \\
g N.T./m²/d & 0.53 & 1.14 & 1.11 & 3.62 \\
g P. T./m²/d & 0.27 & 0.54 & 0.78 & 9.86 \\
\hline CARGA HIDRÁULICA (cm/d) & 5.99 & 7.77 & & \\
\hline
\end{tabular}


sistema de lagunaje convencional, siendo éste de 20 días, mientras en el sistema HRAP se alteraron estos tiempos, ensayando 3, 5 y 10 días, para lo que se realizaron variaciones de caudal mediante la alteración de los volúmenes de agua residual dosificados por bombas de membrana.

Las muestras se tomaron del afluente antes de cada sistema experimental de tratamiento y a la salida de cada secuencia ensayada, así como en el interior de los reactores de los sistemas.

Los parámetros físico-químicos y biológicos estudiados, fueron analizados siguiendo la metodología recomendada por Standard Methods (APHA, 1989).

\section{RESULTADOS Y DISCUSIÓN}

El agua residual que se utilizó como afluente presentó unas características de agua residual urbana muy diluída (Metcalf \& Eddy, 1991; Henze et al, 1997) según las concentraciones medias de diversos parámetros como conductividad eléctrica, sólidos en suspensión, DQO y DBO, posiblemente derivado del efecto de dilución que suponen las muchas infiltraciones y aportaciones al colector de la localidad. Sin embrago, las concentraciones de nutrientes no resultaron tan diluidas en momentos específicos coincidentes con el empleo de abonos y fertilizantes en actividades agrícolas.

Con ello las cargas superficiales aplicadas a los sistemas y reflejadas en la tabla 1 , son menores a las consideradas para un agua residual típicamente urbana (Metcalf \& Eddy, 1991). No obstante, esta es la tónica de las aguas residuales en los pequeños y medianos municipios del noroeste español (Martino, 1998)

Los rendimientos medios de eliminación obtenidos en los parámetros más importantes en el tratamiento del agua residual por los dos sistemas (Sólidos, DQO, DBO y nutrientes), se ofrecen en la tabla 2. Ambos sistemas consiguen un efluente de calidad y admisible para su vertido a un cauce receptor según los requisitos de la legislación vigente. Sin embargo, se aprecia una mayor eficacia del sistema HRAP frente al sistema convencional.
La materia orgánica generada por el sistema HRAP se presenta mayoritariamente en forma particulada debido a los sólidos generados, que a su vez son atribuibles a los valores de concentración de algas, algo que sucede en menor medida en el sistema de lagunaje. La fracción soluble de la materia orgánica y fácilmente biodegradable se genera en menor medida en el sistema HRAP, dada la transformación de la misma durante el proceso en materia orgánica en forma de partículas o microalgas. La menor biodegradabilidad de la materia orgánica del efluente se atribuye a la naturaleza celulósica de las paredes celulares de las microalgas presentes en el líquido de mezcla. La relación de la materia orgánica con los sólidos generados por los sistemas, se correlaciona de forma altamente significativa en el caso del sistema HRAP y sólo en parte en el sistema de lagunaje convencional con las formas totales de la DQO y DBO,.

La eliminación de materia orgánica, tanto medida como DQO o como DBO, teniendo en cuenta las fracciones totales y disueltas, fue superior en el sistema de HRAP frente al lagunaje y dentro del primer sistema, la mayor eficacia se obtuvo para un TRH de 3 días. Estos rendimientos son similares a los obtenidos por otros autores entre los que se destacan los realizados por García, J. (1996) en un sistema experimental de

Table 2. Rendimientos medios de eliminación (\%) obtenidos en los principales parámetros analizados en los sistemas. Mean depuration efficiency $(\%)$ calculated from main variables analysed in both systems (i e. HRAP and natural lagooning).

\begin{tabular}{lcccc}
\hline & & HRAP & & LAGUNA.JE \\
\hline & $\mathbf{1 0}$ & $\mathbf{5}$ & $\mathbf{3}$ & $\mathbf{2 0}$ \\
\hline DQO & 63.95 & 50.74 & 68.54 & 44.18 \\
DBO, & 77.78 & 69.94 & 84.57 & 68.95 \\
$\mathrm{SST}^{-}$ & 0 & 2 & 1 & 8.49 \\
$\mathrm{NO}_{3}{ }^{-}$ & 26.12 & 34.76 & 52.30 & 38.42 \\
$\mathrm{NH}_{4}^{+}$ & 82.30 & 89.5 & 90.46 & 89.31 \\
$\mathrm{N.T.K}^{+}$ & 33.42 & 14.12 & 7.84 & 27.02 \\
PO4 $_{3}{ }^{-}$ & 86.52 & 67.94 & 65.61 & 61.18 \\
P.T. & 85.44 & 73.05 & 78.46 & 68.06 \\
\hline
\end{tabular}


HRAP de iguales características de diseño al utilizado en el estudio, si bien operando con diferentes cargas y en distinto gradiente climático además de realizar tratamiento de la biomasa producida. También son semejantes a los encontrados por Oswald (1991) en un sistema integral avanzado de lagunas en Sta. Helena (EEUU). Otros autores obtuvieron rendimientos mayores en torno al $90 \%$ en DBO, y del $50 \%$ en DQO (Shelef et al, 1980; Azov \& Shelef, 1982; Abeliovich, 1986).

El cálculo de los rendimientos de eliminación de materia orgánica, se ha realizado en base a los resultados observados para los efluentes sobre muestra filtrada, lo que expresa la eficacia de eliminación en materia orgánica disuelta. En otros sistemas de HRAP (El Halouani, 1990) como en el que ha sido estudiado, no se contempla la separación final de los efluentes de la materia en suspensión y los resultados son inferiores en estos parámetros: entre un $15 \%$ a un $20 \%$ de rendimiento de eliminación. García (1996) observó rendimientos en DQO sobre el 35\% sobre muestra total y $68 \%$ sobre muestra filtrada.

Respecto a los sólidos suspendidos y su eliminación, según lo anteriormente expresado y teniendo en cuenta que no se efectúa tratamiento posterior del efluente de los reactores de los sistemas, los rendimientos obtenidos son muy bajos y mayores en el sistema convencional respecto al HRAP, sin duda debido a la mayor producción de biomasa en este último sistema. Se entiende que la evolución de los sólidos, no es en este caso un dato de la eliminación de los mismos, sino mas bien una estimación de su producción.

La eliminación de nutrientes fue desigual. Se obtuvo un rendimiento medio en nitrógeno total mayor en el sistema de lagunaje convencional respecto al HRAP, si bien dentro de este Último la eficacia en el mayor de los tiempos de retención ensayados fue superior, aunque con un amplio rango de variación, respecto al lagunaje. Los resultados globales son inferiores a los encontrados por otros autores en estudios semejantes (Shelef et al, 1980; Fallowfield \& Garret, 1985; Moersidik, 1992; García, 1996). La eficacia media de eliminación no supera el $40 \%$, al igual que lo observado por El Halouani et al (1993) en sistemas de HRAP sin tratamiento posterior de la biomasa producida ( $28 \%$ de media anual).

El rendimiento de eliminación de nitrato, lo que nos da una idea de la posible nitrificación producida en el sistema y la asimilación de compuestos oxidados del nitrógeno, se observó mayor en el HRAP operando a TRH de 3 días, seguido del sistema de lagunaje. Esta tendencia se mostró contraria a la eliminación observada de NTK en cuanto a que es mayor a menor TRH para el sistema HRAP. Ello tiene explicación debido a que los valores de concentración en los efluentes del nitrógeno orgánico evolucionan en el mismo sentido, incrementándose a menores tiempos de residencia, con lo que la vía de asimilación por la biomasa de compuestos oxidados se mostró más efectiva a menores TRH.

La eliminación de nitrógeno amoniacal fue semejante en los dos sistemas e independiente de las condiciones operacionales ensayadas y muy relacionado con los altos niveles de $\mathrm{pH}$ registrados (rangos: sistema de lagunaje: 7.8 - 11.5; sistema HRAP: 7.9 - 10.7) que siendo inferiores a 9 es la forma ionizada del amonio $\left(\mathrm{NH}_{4}^{+}\right)$la que predomina y a $\mathrm{pH}$ superior a 9 domina la forma no ionizada (Moersidik, 1993). Además en el caso del HRAP se ve favorecida la volatilización del amoniaco por la agitación del líquido de mezcla y el consiguiente incremento de capacidad de transferencia que ello supone (Shpirt, 1981). Las posibles vías para su eliminación son la nitrificación del ion amonio y la volatilización del amoníaco así como la asimilación por los microorganismos.

La eliminación media de fósforo total fue elevada en todos los casos si se tiene en cuenta la relación entre muestra total y filtrada, superior al $70 \%$ y semejante a los observados por Shelef $\&$ Azov (1980) y Fallowfield \& Garret (1985) si bien estos autores al igual que García (1996) contemplan la eliminación ulterior de la biomasa producida. En el caso de no contemplarse esta eliminación, como ocurre en El Halouani et al (1993), los rendimientos son inferiores (del orden del 15\%) en el balance neto del fósforo total. Se obtuvieron mejores resultados para el HRAP y 
dentro de este sistema parece observarse una mejor eliminación cuanto mayor es el tiempo de permanencia, dato que se corrobora con las eficacias encontradas para el fósforo inorgánico soluble expresado como ortofosfato. El incremento de fosforo orgánico particulado en los efluentes de los sistemas, es mayor en el HRAP y dentro del mismo sistema, más elevado a mayores tiempos de residencia.

La importancia de los nutrientes como factor limitante en el crecimiento de las microalgas, que en los casos que nos ocupan y, como ya se ha comentado anteriormente, se relaciona con la biomasa producida, ha sido discutida en numerosas ocasiones. Se ha supuesto en el caso de los sistemas de lagunaje que los aportes de nutrientes por el agua residual cruda de carácter urbano, no existe limitación nutricional respecto el carbono, nitrógeno y fósforo (Abeliovich \& Weismann, 1978). Sin embargo y como se destacó anteriormente, el carácter del agua residual utilizada como afluente es de muy baja concentración en nutrientes y muy diluida, fundamentalmente en carbono. Según Oswald (1986) y Abeliovich (1986) en este tipo de aguas diluidas el carbono es el principal nutriente que limita el crecimiento de las microalgas, ya que su proporción relativa es inferior a los otros nutrientes.

En los sistemas de lagunaje, las bacterias tienen su fuente de carbono en la degradación heterotrófica de la materia orgánica del agua residual; sin embargo, las microalgas pueden obtener el carbono mediante tres fuentes alternativas (Azov et al, 1982): el dióxido de carbono obtenido por la degradación de la materia orgánica, los bicarbonatos presentes en el agua residual y finalmente el dióxido de carbono atmosférico disuelto en el agua.

La mayoría de las microalgas obtienen el carbono por la vía indirecta de la degradación bacteriana de la materia orgánica y asimilación del CO,. Azov et al (1982) observaron que cuando la carga orgánica del agua residual es baja, esta fuente de carbono derivada de la respiración no es suficiente a los requerimientos del sistema y el cultivo depende de la asimilación de carbono atmosférico, ya que el suministro artificial de dióxido de carbono a sistemas con estas deficiencias, corrige la limitación. Además refuerza esta hipótesis el hecho de que el suministro de bicarbonato a sistemas con las deficiencias mencionadas no suple la limitación de este elemento. Otros autores como Gutterman \& Ben Yaakov (1987) observaron que el flujo atmosférico de carbono es insuficiente para mantener una producción primaria acorde con las posibilidades de los sistemas.

La carga orgánica superficial aplicada a los sistemas para que no exista limitación de carbono por la vía de la degradación bacteriana, ha de ser superior a los $400 \mathrm{~kg} \mathrm{DBO}_{5}$ /ha.día (Azov et al, 1982). Estos valores no se alcanzan en los sistemas estudiados ni en las estrategias operacionales seguidas, ya que como máximo se alcanza el valor medio de $19.2 \mathrm{~kg} \mathrm{DBO}_{5} /$ ha día el en HRAP con TRH de 3 días y en el lagunaje convencional $6.6 \mathrm{~kg} \mathrm{DBO}_{5} /$ ha.día. Se manifiesta en esta limitación la estrategia seguida por los autores de procedencia israelita en la que la producción de biomasa en uno de los requisitos a considerar en los sistemas de tratamiento basados en microalgas, además del tratamiento secundario del agua residual. Así cabe apreciar que los sistemas estudiados por nosotros son sistemas de tratamiento de agua residual con un déficit de carbono con respecto a otros nutrientes.

La producción de biomasa en un sistema que se basa en el crecimiento de microalgas, puede ser estimada en función de las concentraciones de clorofila que se dan en el reactor, ya que su concentración supone en promedio el $1.5 \%$ del peso seco de la materia orgánica (libre de cenizas) del alga (Margalef, 1986; APHA, 1989). Basándose en este concepto, se estimaron unas producciones globales de algas en los sistemas de $32.29 \mathrm{~g} / \mathrm{m}^{3}$ en peso seco para el sistema de HRAP y de 9.41 $\mathrm{g} / \mathrm{m}^{3}$ en peso seco para el sistema de lagunaje convencional.

Teniendo en cuenta las superficies y tiempos de retención que se emplearon en cada planta experimental se obtuvieron valores de producción de $144.79 \mathrm{~kg} / \mathrm{ha}$ año para el HRAP y $18.57 \mathrm{Kg} / \mathrm{ha}$ año para el lagunaje. Con lo cual los valores de producción arrojados por el sistema de HRAP son 


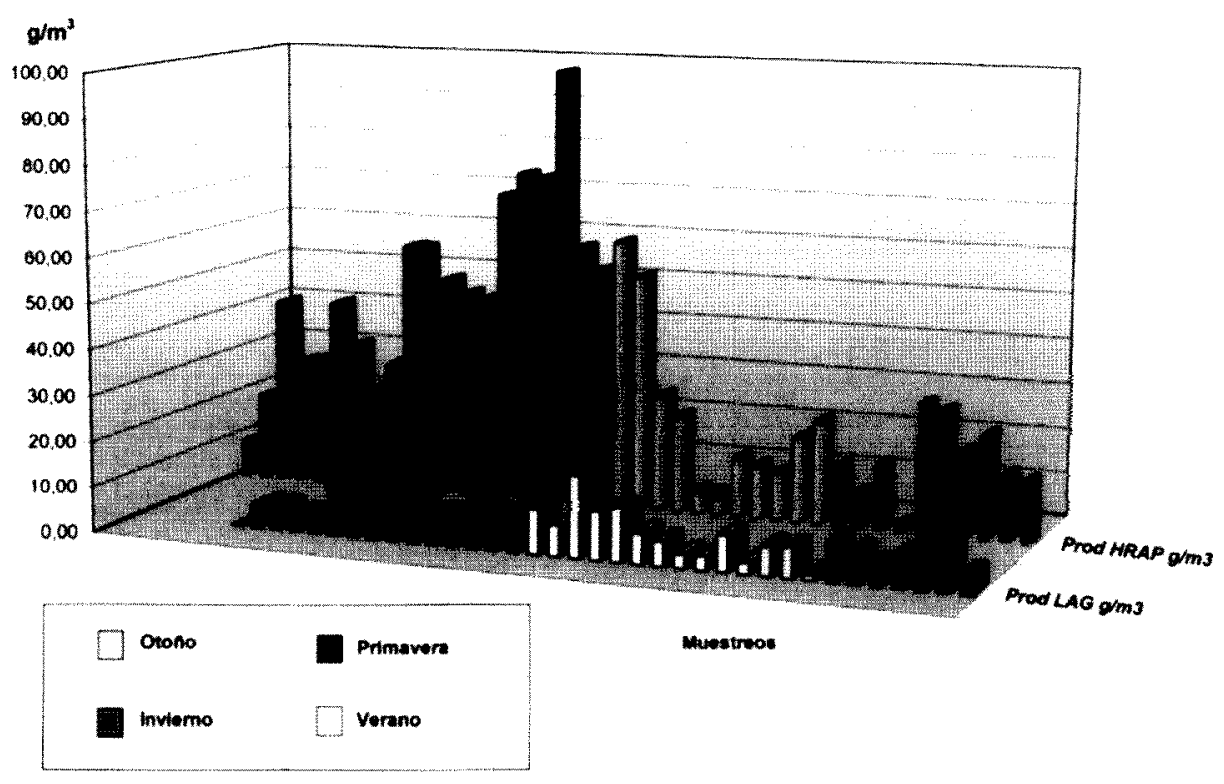

Figura 1. Evolución estaciona1 de la producción estimada de biomasa expresada en $\mathrm{g} / \mathrm{m}^{3}$ para los sistemas de HRAP y lagunaje convencional. Estimated algal biomass $\left(\mathrm{g} \mathrm{m}^{-3}\right)$ in the HRAP and natural lagoons.

muy superiores al sistema convencional, incluso disponiendo este último de mayor superficie y mayor tiempo de permanencia del agua en el reactor. Sin embargo todos estos valores son inferiores a los citados en bibliografía (Shelef \& Soeder, 1980; Azov et al 1982; Ritchmond, 1986; Borowitza \& Borowitza, 1988; Oswald, 1988, García, 1996), posiblemente como consecuencia de que los sistemas no se encuentran completamente eutrofizados y las bajas cargas orgánicas y de nutrientes que aporta el agua utilizada como afluente, fundamentalmente la limitación que supone el carbono, como ha sido comentado.

En la figura 1 se expresa gráficamente la evolución de la producción estimada para cada planta experimental en las distintas épocas estacionales. Se observa que la mayor producción estimada se obtuvo en la primavera para el HRAP o inmediatamente antes de la misma para el lagunaje convencional. Además se obtuvieron mayores niveles de producción en los muestreos correspondientes al invierno, que los observados durante el verano.

En el sistema HRAP la dinámica poblacional de las especies del fitoplancton contribuyó de forma determinante a esta producción de biomasa. Como se observa en la tabla 3 la especie mayoritaria en las diferentes estaciones fue Monoraphidium contortum Sus "blooms" se mostraron permanentes durante todo el estudio, y fueron determinantes junto a las especies acompañantes de la biomasa producida. Además, un aspecto importante es que se trata de especies con capacidad de sedimentación, o bien capaces de asociarse en flóculos que permitan su simple decantación física, aspecto éste importante en un sistema de las características del estudiado y de la calidad admisible del efluente para ser vertido en un cauce natural receptor.

Atendiendo a la taxonomía, la clase con mayor abundancia fue la de clorofíceas o algas verdes, seguida por la abundancia de flagelados fotosintéticos. Dentro de la clase Chlorophyceae la mayor abundancia fue del género Monoraphidium sp, seguido por las especies del género Scenedesmus sp y Chlorella $s p$.

La evolución y dinámica de las especies se observó determinada por la estacionalidad más que por la variación de las condiciones operacionales del sistema. Se observaron valores máxi- 
Table 3. Densidad de la población de algas (cells ml-1) en el sistema HRAP. Algal population density (cells ml-1) in the HRAP system lagoon.

\begin{tabular}{|c|c|c|c|c|c|}
\hline Clase & Género & Media & Max. & Min. & Desv. \\
\hline Cyanophyceae & Microcystis & 1492 & 4240 & 0 & 1598 \\
\hline \multirow[t]{2}{*}{ Euglenophyceae } & Euglena & 236 & 707 & 0 & 353 \\
\hline & Phacus & 471 & 2120 & 0 & 935 \\
\hline Cryptophyceae & Cryptomonas & 79 & 707 & 0 & 236 \\
\hline \multirow[t]{7}{*}{ Chlorophyceae } & Chlamydomonas & 314 & 1413 & 0 & 513 \\
\hline & Chlorogoniurn & 1411 & 6360 & 0 & 2291 \\
\hline & Carteria & 79 & 707 & 0 & 236 \\
\hline & Chlorococcum & 628 & 4947 & 0 & 1636 \\
\hline & Chlorella & 43198 & 228253 & 0 & 72068 \\
\hline & Scenedesmus & 166229 & 448733 & 24027 & 140781 \\
\hline & Monoraphidium & 780829 & 2055693 & 234613 & 509319 \\
\hline \multirow[t]{4}{*}{ Bacillariophyceae } & Cymbella & 16398 & 42400 & 2120 & 14408 \\
\hline & Nitzschia & 2720 & 7523 & 0 & 2736 \\
\hline & Navicula & 236 & 2120 & 0 & 707 \\
\hline & Diatoma & 2979 & 21200 & 0 & 6869 \\
\hline \multirow[t]{2}{*}{ Otros } & Flagelados fotosint. & 6988 & 52293 & 0 & I7347 \\
\hline & "Indeterm." & 38862 & 344853 & 0 & 114750 \\
\hline TOTAL (células/ml) & & 1063224 & 2491707 & 645893 & 557663 \\
\hline
\end{tabular}

mos en primavera con una formación de $1.40 .10^{6}$ células $/ \mathrm{m}^{3}$ en primavera de las cuales el $82.66 \%$ fue Monoraphidium contortum y el resto (17.34\%) correspondió a los 11 géneros acompañantes más los flagelados. De entre todos ellos destacó el género Scenedesmus sp. que supuso el $13.54 \%$ del total. Esta estructura fue prácticamente mantenida a lo largo del estudio, si bien las cantidades o abundancias celulares medias en otras épocas fueron menores. Así en verano se alcanzó el valor medio de $\mathrm{I} .02 \cdot 10^{6}$ células $/ \mathrm{m}^{3}$ con un total de 10 géneros mayoritarios y en invierno $0.77 \cdot 10^{6} \mathrm{cel} / \mathrm{m}^{3}$ con 11 especies con menor abundancia relativa frente a la dominante.

Se observaron alternancias estacionales detectadas para el género Scenedesmus sp, del que se determinaron 3 especies diferentes: S. quadricau$d a, S$. obliquus y S. quadriespina. Otras alternancias se manifiestaron entre géneros así, Cryptomonas sp sólo apareció en invierno al igual que Carteria sp. y al comienzo del estudio, Clha- mydomonas sp desapareció en verano, Chlorella $s p$. fue más abundante en verano. Mycrocystis $s p$. se mantuvo hasta primavera pero luego desaparecio a partir del verano. Las diatomeas fueron más constantes si bien apareció Diatoma sp. a finales del estudio.

La dinámica poblacional de las especies del fitoplancton en el lagunaje convencional , evolucionó a lo largo del estudio de forma semejante al valor de producción estimada y detallada en la tabla 4. De esta forma se cuantificaron una media del número de células por volumen de $0.3 \cdot 10^{6}$ $\mathrm{cel} / \mathrm{m}^{3}$ en el periodo invernal, muy superior a los $0.07 .10^{6} \mathrm{cel} / \mathrm{m}^{3}$ en primavera y $0.04 \cdot 10^{6} \mathrm{cel} / \mathrm{m}^{3}$ en verano. La evolución de especies en la época del invierno se vio dominada por Chlorella sp. que representó el $53.72 \%$ del total de individuos, seguida por especies del género Cryptomonas $\mathrm{sp}$. (C. erosa y C. ovata), que alcanzaron en esta época el $31.66 \%$. Además de los ya citados, se determinaron un total de 12 taxones más, lo que 
Table 4. Densidad de la población de algas (cells $\mathrm{ml}^{-1}$ ) en el sistema de lagunaje convencional. Algalpopulation density (cells $\mathrm{ml}^{-1}$ ) in the natural lagoon system.

\begin{tabular}{|c|c|c|c|c|c|}
\hline Clase & Género & Media & Max. & Min. & Desv. \\
\hline Cyanophyceae & Microcystis & 1837 & 9187 & 0 & 3393 \\
\hline Euglenophyceae & Euglena & 1084 & 5653 & 0 & 2079 \\
\hline Cryptophyceae & Cryptomonas & 71636 & 294680 & 2120 & 107226 \\
\hline Chlorophyceae & $\begin{array}{l}\text { Chlamydomonas } \\
\text { Chlorogonium } \\
\text { Chlorococcum } \\
\text { Chlorella } \\
\text { Scenedesmus } \\
\text { Monoraphidium }\end{array}$ & $\begin{array}{r}1373 \\
686 \\
202 \\
75916 \\
40 \\
182\end{array}$ & $\begin{array}{r}7067 \\
3533 \\
1413 \\
253693 \\
283 \\
707\end{array}$ & $\begin{array}{r}0 \\
0 \\
0 \\
942 \\
0 \\
0\end{array}$ & $\begin{array}{r}2627 \\
1291 \\
534 \\
113864 \\
107 \\
279\end{array}$ \\
\hline Bacillariophyceae & $\begin{array}{l}\text { Nitzschia } \\
\text { Navicula } \\
\text { Diatoma }\end{array}$ & $\begin{array}{r}680 \\
949 \\
40\end{array}$ & $\begin{array}{r}2120 \\
5276 \\
283\end{array}$ & $\begin{array}{l}0 \\
0 \\
0\end{array}$ & $\begin{array}{r}799 \\
1941 \\
107\end{array}$ \\
\hline Otros & $\begin{array}{l}\text { Flagelados fotosint. } \\
\text { "Indeterm." }\end{array}$ & $\begin{array}{r}20588 \\
256\end{array}$ & $\begin{array}{r}73493 \\
1413\end{array}$ & $\begin{array}{l}0 \\
0\end{array}$ & $\begin{array}{r}26580 \\
521\end{array}$ \\
\hline TOTAL (células/mI) & & 175469 & 603493 & 21765 & 210666 \\
\hline
\end{tabular}

representó el momento estacional con mayor diversidad. En primavera el total de taxones determinados fue de 12 , sin embargo la dominancia citada del género Chlorella sp. fue sustituida por la de Cryptomonas sp. representando la primera solamente el $3.46 \%$ del total y la segunda el $74.35 \%$. La diversidad en verano fue menor que en otras estaciones, al igual que sucedió con su número o abundancia, detectándose la presencia de 6 "taxa", siendo el más dominante la especie Cryptomonas ovata que supuso el $82.5 \%$ del total para esta estación.

\section{CONCLUSIONES}

El sistema HRAP genera una mayor cantidad de biomasa algal.

La estratificación del sistema convencional condiciona su biomasa y la alternancia en la abundancia y dominancia de las especies.

Se genera mayor proporción de materia orgánica particulada en el sistema HRAP y ésta está asociada a la producción de biomasa.

La decantación de la biomasa generada por los sistemas se ve favorecida por la composición específica de la misma, siendo más factible en el sistema HRAP.

El efecto de dilución del agua utilizada como afluente condiciona y limita el crecimiento, primeramente por la fuente de carbono y posteriormente en las necesidades de otros nutrientes $(\mathrm{N}$ y $\mathrm{P})$.

Los rendimientos obtenidos son adecuados a las exigencias legales sobre la calidad de los efluentes y son mayores en el sistema HRAP.

\section{BIBLIOGRAFÍA}

ABELIOVICH, A. 1986. Algae in wastewater oxidation ponds. In: Handbook of Microalgal Mass Culture. A. Richmond (ed.): 331-338. CRC Press. Boca Raton.

ABELIOVICH, A. D. WEISMAN. 1978. Role of heterotrophic nutrition in growth of the alga Scenedesmus obliquus in high rate oxidation 
ponds. Applied and Environmental Microbiology, 35 (1): 31-37

APHA-AWWA-WPCF. 1989. Standard Methods for the Examination of Water and Wastewater. Ed. Clesceri, L.S.; A.E. Greenberg \& R.R. Trussell. 17 th Edition. Baltimore. Marylan

AZOV, Y. \& G. SHELEF. 1982. Operation of high-rate oxidation ponds: theory and experiments. Wat. Res., 16: 1153-1160.

AZOV, Y., G. SHELEF AND N. NARKIS. 1982. Effect of hard detergents on algae in a high rate oxidation pond. Applied and Environmental Microbiology, 43 (2): 491-492.

AZOV, Y., G. SHELEF \& R. MORAINE. 1982. Carbon limitation of biomass production in high rate oxidation ponds. Biotechnol. Bioeng., 24: 579594.

BOROWITZKA, M.A. \& L. J. BOROWITZKA, L. J. (ed). 1988. Microalgal Biotechnology. Cambridge University Press. Cambridge. 477 pp.

EL HALOUANI, H. 1990. Lagunage a Haut rendement: caracterisation physico-chimique de l'ecosysteme etude de son aptitude a I'elimination de l'azote et du phosphore dans I'epuration de eaux usees. Tesis doctoral, Montpelier (France). $154 \mathrm{pp}$

EL HALOUANI, H., B. PICOT, C. CASELlAS, G. PENA \& J. BONTOUX. (1993). Elimination de l'azote et du phosphore dans un lagunage á haut rendement. Revue des Sciences de l'Eau, 6: 47-61.

FALLOWFIELD, H. J. \& M. K. GARRET. 1985. The treatment of wastes by algal culture. Journal of Applied Bacteriology, Symposium Supplement: 187-205.

GARCÍA, J. 1996. Eliminació de materia orgànica $i$ nutrients en llacunes d'alt rendiment. Tesis doctoral. Universidad de Barcelona. 301 pp.

GUTTERMAN, H. \& S. BEN-YAAKOV. 1987. Exchange rates of 02 and $\mathrm{CO} 2$ between an algal culture and atmosfere. Wat. Res., 21:25-34.

HENZE, M., P. HARREMOES, J. LA COUR JANSEN, E. ARVIN. 1997. Wastewater treatmnet. Springer, Berlin.

MARA, D. D. \& S. CAIRNCROSS. 1990. Directrices para el uso sin riesgos de aguas residuales y excretas en agricultura y acuicultura. Medidas de protección de la salud pública. OMS, Ginebra, $213 \mathrm{pp}$

MARGALEF, R. 1982. Ecología. Editorial Omega. Barcelona. $951 \mathrm{pp}$

MARTINO, J. 1998. Futuros planes de actuación para el saneamiento de pequeños municipios.: 155-174. En: Libro Blanco sobre calidad de agua en el
Bierzo (León, Spain). J.M. González and A. Cabo. Universidad de León. León.

METCALF \& EDDY, INC. 1991. Wastewater Engineering, Treatment, Disposal and Reuse - $3^{\text {rd }}$ Ed.. McGraw-Hill Inc. New York. 1334 pp.

MOERSIDIK, S. S. 1992. Traitement des eaux usees par lagunage a haut rendement: Gestion del'ecosysteme et comparaison des performances epuratories avec un lagunaje traditionnel. These Doctorel, Universite de Montpellier I. $154 \mathrm{pp}$

MOPT. 1992. Informe de la comisión delegada del Gobierno para asuntos económicos sobre la depuración de aguas residuales urbanas en España y las implicciones de la aplicación de la Directiva 91/271/CEE. Servicio de documentación y Publicaciones Secretaría General de Medio Ambiente. Ministerio de Obras Públicas y Transportes.

MUJERIEGO, R. 1990. Riego con agua residual municipal regenerada. Manual práctico. Universidad Politécnica de Cataluña, 481 pp.

OSWALD, W. J. (ed.). 1986. A syllabus on Waste Pond Fundamentals University of California. Berkeley. $187 \mathrm{pp}$.

OSWALD, W. J. 1988. Large-scale algal culture systems (engineering aspects). In: Microalgal Biotechnology. M.A. Borowitzka \& L. J. Borowitzka (eds.): 357-394. Cambridge University Press. Cambridge.

OSWALD, W. J. 1988. Micro-algae and waste-water Treatment. In: Microalgal Biotechnology. M.A. Borowitza \& L.J. Borowitzka. (eds): 305-328. Cambridge University Press.

OSWALD, W. J. 1991. Introduction to advanced integrated wastewater ponding systems. Wat. Sci. Tech., 24: 1-7.

OSWALD, W. J. \& H. B. GOTAAS. 1957. Photosynthesis in sewage treatment. Trans. Arn. Soc. Civ. Eng., 122: 73-105.

OSWALD, W. J., H. B. GOTAAS, C. G. GOLUEKE \& W. R. KELLEN. 1957. Algae in waste treatment. Sewage Ind. Wastes, 29: 437-457.

PAPADAKIS, P. 1961. Climatic tables for the word. Buenos Aires.

RICHMOND, A. (ed). 1986. Handbook of Microalgal mass culture. CRC press. Boca Raton, Florida. $528 \mathrm{pp}$.

SHELEF, G. 1987. Conclusions. 6. High-rate algal ponds. Wat. Sci. Tech., 19: 396-397.

SHELEF, G. \& Y. AZOV. 1987. High-rate oxidation ponds: the israeli experience. Wat. Sci. Tech., 19: 249-255. 
SHELEF, G \& C. J. SOEDER. (eds). 1980. Algae Biomass. Production and use. Elsevier/ North Holland Biomedical1 Press. Amsterdam. $852 \mathrm{pp}$.

SHELEF, G., R. MORAINE \& G. ORON. 1980. Algal mass production as an integral part of waste treat- ment \& reclamation system. In: Algae Biomass. G. Shelef \& J. Soeder (eds.): 163-190. Elsevier/North Holland Biomedical Press. Amsterdam.

SIERRA, J y L. PEÑALVER. 1989. La reutilización de las agua residuales. Acondicionamiento y uso. CEDEX, 216 pp. 